\title{
A tertiary center experience of multiple myeloma patients with COVID-19: lessons learned and the path forward
}

Bo Wang ${ }^{1 \dagger}$, Oliver Van Oekelen ${ }^{1 \dagger}$, Tarek H. Mouhieddine ${ }^{2}$, Diane Marie Del Valle ${ }^{1}$, Joshua Richter ${ }^{1}$, Hearn Jay Cho ${ }^{1}$, Shambavi Richard ${ }^{1}$, Ajai Chari ${ }^{1}$, Sacha Gnjatic ${ }^{1}$, Miriam Merad ${ }^{1,3}$, Sundar Jagannath', Samir Parekh ${ }^{1}$ and Deepu Madduri ${ }^{1 *}$ (D)

\begin{abstract}
Background: The COVID-19 pandemic, caused by SARS-CoV-2 virus, has resulted in over 100,000 deaths in the USA. Our institution has treated over 2000 COVID-19 patients during the pandemic in New York City. The pandemic directly impacted cancer patients and the organization of cancer care. Mount Sinai Hospital has a large and diverse multiple myeloma (MM) population. Herein, we report the characteristics of COVID-19 infection and serological response in MM patients in a large tertiary care institution in New York.

Methods: We performed a retrospective study on a cohort of 58 patients with a plasma-cell disorder (54 MM, 4 smoldering MM) who developed COVID-19 between March 1, 2020, and April 30, 2020. We report epidemiological, clinical, and laboratory characteristics including the persistence of viral detection by polymerase chain reaction (PCR) and anti-SARS-CoV-2 antibody testing, treatments initiated, and outcomes.

Results: Of the 58 patients diagnosed with COVID-19, 36 were hospitalized and 22 were managed at home. The median age was 67 years; $52 \%$ of patients were male and 63\% were non-White. Hypertension (64\%), hyperlipidemia (62\%), obesity (37\%), diabetes mellitus (28\%), chronic kidney disease (24\%), and lung disease (21\%) were the most common comorbidities. In the total cohort, 14 patients (24\%) died. Older age (> 70 years), male sex, cardiovascular risk, and patients not in complete remission (CR) or stringent CR were significantly $(p<0.05)$ associated with hospitalization. Among hospitalized patients, laboratory findings demonstrated elevation of traditional inflammatory markers (CRP, ferritin, D-dimer) and a significant $(p<0.05)$ association between elevated inflammatory markers, severe hypogammaglobulinemia, non-White race, and mortality. Ninety-six percent (22/23) of patients developed antibodies to SARS-CoV-2 at a median of 32 days after initial diagnosis. The median time to PCR negativity was 43 (range 19-68) days from initial positive PCR.

(Continued on next page)
\end{abstract}

\footnotetext{
* Correspondence: deepu.madduri@mountsinai.org

${ }^{\dagger}$ Bo Wang and Oliver Van Oekelen contributed equally to this work.

'Tisch Cancer Institute, Icahn School of Medicine at Mount Sinai, 10 East 102nd Street, 6th Floor, New York, NY 10029, USA

Full list of author information is available at the end of the article
}

(c) The Author(s). 2020 Open Access This article is licensed under a Creative Commons Attribution 4.0 International License, which permits use, sharing, adaptation, distribution and reproduction in any medium or format, as long as you give appropriate credit to the original author(s) and the source, provide a link to the Creative Commons licence, and indicate if changes were made. The images or other third party material in this article are included in the article's Creative Commons licence, unless indicated otherwise in a credit line to the material. If material is not included in the article's Creative Commons licence and your intended use is not permitted by statutory regulation or exceeds the permitted use, you will need to obtain permission directly from the copyright holder. To view a copy of this licence, visit http://creativecommons.org/licenses/by/4.0/ The Creative Commons Public Domain Dedication waiver (http://creativecommons.org/publicdomain/zero/1.0/) applies to the data made available in this article, unless otherwise stated in a credit line to the data. 


\begin{abstract}
(Continued from previous page)
Conclusions: Drug exposure and MM disease status at the time of contracting COVID-19 had no bearing on mortality. Mounting a severe inflammatory response to SARS-CoV-2 and severe hypogammaglobulinemia was associated with higher mortality. The majority of patients mounted an antibody response to SARS-CoV-2. These findings pave a path to the identification of vulnerable MM patients who need early intervention to improve outcomes in future outbreaks of COVID-19.
\end{abstract}

Keywords: Multiple myeloma, Smoldering multiple myeloma, COVID-19, SARS, SARS-Cov-2, New York, Pandemic

\section{Introduction}

The coronavirus disease 2019 (COVID-19) pandemic, caused by severe acute respiratory syndrome coronavirus2 (SARS-CoV-2), represents a world-wide public health crisis. Patient care has been drastically altered, primarily in epidemic, urban areas. As of May 22, 2020, New York City had nearly 200,000 confirmed cases of COVID-19 with over 16,000 deaths and a patient death rate of $21 \%$ [1], with cancer patients comprising about $8 \%$ of all COVID19 fatalities in the state of New York (https://covid1 9tracker.health.ny.gov/). Mount Sinai Hospital, a tertiary care center in New York City, has treated over 2000 admitted COVID-19 patients thus far. At our cancer center, we actively care for a large and particularly diverse population of over 3000 multiple myeloma (MM) patients. Like many other centers in the region and the world, clinical care at our institution has seen significant changes in an attempt to mitigate the spread of SARS-CoV-2 to vulnerable cancer patients receiving treatment. Balancing the competing risks of treatment delay or alteration versus infection is essential and depends upon understanding the clinical profile of COVID-19 in this vulnerable population.

Limited studies describing the impact of COVID-19 both in the USA [2] and abroad [3-6] suggest a higher risk of hospitalization and poor outcomes including death in certain subsets of cancer patients. The effect of COVID-19 on patients with MM, the second most common hematological malignancy, is of particularly great concern due to immunosuppression associated with the disease, and at this time remains incompletely understood. MM is a plasma cell malignancy, diagnosed at a median age around 70 years in patients often with multiple comorbidities [7]. MM is associated with both cellular and humoral immune dysfunction and causes a state of generalized immune suppression, leaving patients especially vulnerable to infections $[8,9]$.

In contrast to the reported immunosuppressive nature of MM, COVID-19 infection has demonstrated a propensity for triggering an uncontrolled immune inflammatory cascade [10-12] that bears resemblance to cytokine release syndrome (CRS) seen in patients treated with chimeric antigen receptor (CAR)- $T$ cells and bispecific antibodies $[13,14]$. Inflammatory markers and cytokines, including C-reactive protein (CRP), ferritin, and interleukin (IL)-6, have been significantly elevated in multiple cohorts of patients infected with COVID-19 [15-18].

We aimed to characterize the population of MM patients at our institution who developed COVID-19 in the epicenter of the pandemic in the USA. To address this, we retrospectively analyzed a cohort of $58 \mathrm{MM}$ and smoldering MM (SMM) patients treated at the Mount Sinai Hospital who were diagnosed with COVID-19 between March 1 and April 30, 2020. We have identified several demographic characteristics and comorbidities associated with hospitalization and elevation of certain inflammatory markers associated with increased mortality as described below.

\section{Methods}

Study design, inclusion criteria, and data collection

The study was designed from a register of patients with SMM and MM in any phase of the response, currently receiving treatment or follow-up at the Mount Sinai Hospital. All patients with a confirmed or presumptive diagnosis of COVID-19 between March 1, 2020, and April 30 , 2020, were considered potentially relevant. Infection with SARS-CoV-2 was confirmed by Roche Cobas 6800 polymerase chain reaction (PCR) in patients that were treated at the Mount Sinai Hospital. For patients admitted to other hospital systems, inclusion was based on external reporting and follow-up testing confirmation. Similarly, outpatients that reported a positive COVID-19 test to our clinic (e.g., over the phone) were included in the analysis, awaiting collection of their formal test results. Anti-SARSCoV-2 antibody testing was performed using an anti-IgG assay developed at Mount Sinai Health System Department of Pathology in collaboration with the Icahn School of Medicine at Mount Sinai Department of Microbiology under a Food and Drug Administration (FDA) Emergency Use Authorization. We reviewed clinical charts, nursing records, laboratory findings, and radiological images for patients and obtained demographic data from the electronic medical records. Plasma levels of selected inflammatory cytokines, including IL-1 $\beta$, IL-6, IL-8, and tumor necrosis factor- $\alpha$ (TNF- $\alpha$ ), were assessed using the ELLA rapid detection enzyme-linked immunosorbent assay (ELISA) microfluidic platform and made available through 
the Mount Sinai data warehouse for hospitalized patients. Treatment response criteria were used as defined by the International Myeloma Working Group (IMWG) [19, 20]. This retrospective study was approved by the institutional review board (IRB) of the Mount Sinai Hospital and is in compliance with the Declaration of Helsinki and International Conference on Harmonization Guidelines for Good Clinical Practice (IRB: GCO\#: 11-1433).

\section{Statistical analysis}

Continuous variables are presented as a median and interquartile range (IQR). Categorical variables are shown as a percentage and an absolute number of patients. Wherever two outcome groups are compared, Fisher's exact test was used to determine significance and odds ratios (ORs) were reported for categorical variables and Mann-Whitney $U$ test was used to determine significance for continuous variables. A two-sided alpha $<0.05$ was considered statistically significant. All statistical analyses were done using $\mathrm{R}$ (version 3.6.1).

\section{Results}

\section{Baseline characteristics}

Our cohort of 58 patients encompassed 52\% males and had a median age of 67 years (IQR: 12.5 years), with $17 \%$ of patients older than 75 years (Table 1). The median body mass index (BMI) was $27.6 \mathrm{~kg} / \mathrm{m}^{2}$ (with $37 \%$ of patients with a BMI $>30 \mathrm{~kg} / \mathrm{m}^{2}$ ). The majority of patients reported being non-White (63\%), with 13 (23\%) patients of African American and 9 (16\%) of Hispanic origin.

The most common comorbidities were hypertension (64\%), hyperlipidemia (62\%), previous or active smoking (37\%), diabetes mellitus type 2 (28\%), chronic kidney disease $(\mathrm{CKD}$, estimated glomerular filtration rate (eGFR) < $60 \mathrm{~mL} / \mathrm{min})(24 \%)$, and lung disease (21\%), including asthma or chronic obstructive lung disease (COPD). Thirty-two (55\%) patients had a high-risk cardiovascular profile (defined as having $\geq 2$ of the conditions: hypertension, hyperlipidemia, and diabetes) and 13 (22\%) had coronary artery disease (CAD) and/or cerebrovascular disease. Seven $(12 \%)$ patients had congestive heart failure. Twelve (21\%) patients were on therapeutic anticoagulation and 34 (59\%) were on aspirin, while 26 (45\%) patients were on an angiotensin-converting enzyme (ACE) inhibitor or angiotensin II receptor blocker (ARB).

\section{Myeloma characteristics}

The cohort included 54 MM and 4 SMM patients (Table 2). The median time from diagnosis to COVID-19 infection was 29.8 months (IQR: 44.2 months). MM patients had a median of 1.5 (IQR: 2) lines of therapy, and 9 (17\%) patients had more than 4 previous lines of treatment. Twenty-two (41\%) patients had a prior autologous stem cell transplant (ASCT).
The median age of patients with and without prior ASCT was 63.5 and 70 years, respectively. Of all patients, 27 (47\%) had an Eastern Cooperative Oncology Group (ECOG) performance status of 0 at the time of COVID-19 infection. The most common myeloma subtype was IgG (59\%) followed by IgA (19\%), with light chain involvement in 33\% of cases. High-risk cytogenetics were present in 22 (39\%) patients, with 18 (33\%) patients having an international staging system (ISS) of 1, while $14(26 \%)$ and $10(19 \%)$ patients had ISS 2 and 3, respectively, at time of MM diagnosis. At the time of SARS-CoV-2 infection, 3 SMM and $8 \mathrm{MM}$ patients were not on therapy. Among the remaining patients, 28 (48\%) patients were being treated with daratumumab, 32 (55\%) patients were on immunomodulatory drugs (IMiDs), 22 (38\%) were on a proteasome inhibitor (PI), 5 (9\%) were on venetoclax, and 30 (52\%) patients were on concomitant corticosteroids.

The disease status at the time of SARS-CoV-2 infection included 15 (26\%) patients in complete response (CR) or stringent CR (sCR), 11 (19\%), 13 (22\%), and 2 (3\%) patients who had a very good partial response (VGPR), partial response (PR), and stable disease (SD), respectively, and 9 (16\%) who had progressive disease (PD). Response status was not evaluable for 8 (14\%) of patients (including 4 SMM patients and 1 newly diagnosed patient).

Biochemical parameters at the last routine clinic visit before presentation with COVID-19 were collected to determine if these steady-state parameters would provide insight into which patients are particularly vulnerable (Table 2). Twenty (35\%) patients had leukopenia $(<4 \times$ $10^{9} / \mathrm{L}$ ) and $7(12 \%)$ lymphocytopenia (grade $3,<0.5 \times$ $\left.10^{9} / \mathrm{L}\right)$ at their last clinic visit. The monoclonal spike (M-spike) was undetectable in 31 (54\%) patients. Median serum IgG level of all patients was $805 \mathrm{mg} / \mathrm{dL}$ (IQR: 736 $\mathrm{mg} / \mathrm{dL})$ and $37 \%(21 / 57)$ of patients had hypogammaglobulinemia $(<700 \mathrm{mg} / \mathrm{dL})$, while $11 \%(6 / 57)$ of patients had severe $(<400 \mathrm{mg} / \mathrm{dL})$ hypogammaglobulinemia. Immunoparesis, defined as a reduction in one or more of the uninvolved immunoglobulins below the lower limit of normal [21], was present in 89\% (51/57) of patients.

\section{Clinical course and biochemical parameters}

The most common reported symptoms among all patients were fever (70\%), cough (65\%), and dyspnea (45\%). Thirty-six patients were admitted at a hospital for inpatient care, 23 of which were admitted at our healthcare system and had both clinical and biochemical parameters available, as shown in Table 3. The median time between self-reported symptom onset and admission was 3 days. Among the 23 patients, 16 (70\%) were febrile, and 11 (48\%) were tachycardic with a heart rate $>100$ beats per minute (bpm) at the time of presentation. Ten $(43 \%)$ patients required immediate oxygen 
Table 1 Demographics and baseline characteristics of patients

\begin{tabular}{|c|c|c|c|c|c|c|c|c|}
\hline \multirow{3}{*}{$\begin{array}{c} \\
\text { Demographics } \\
\text { Age (years) }\end{array}$} & \multirow{2}{*}{\multicolumn{2}{|c|}{$\begin{array}{l}\text { All patients } \\
\boldsymbol{n}=58\end{array}$}} & \multirow{2}{*}{\multicolumn{2}{|c|}{$\begin{array}{l}\text { Patients not } \\
\text { admitted to } \\
\text { hospital } \\
\boldsymbol{n}=22\end{array}$}} & \multirow{2}{*}{\multicolumn{2}{|c|}{$\begin{array}{l}\text { Hospitalized, } \\
\text { discharged alive } \\
\boldsymbol{n}=22\end{array}$}} & \multirow{2}{*}{\multicolumn{2}{|c|}{$\begin{array}{l}\text { Hospitalized, } \\
\text { deceased } \\
\boldsymbol{n}=14\end{array}$}} \\
\hline & & & & & & & & \\
\hline & 67 & {$[12.5]$} & 64 & {$[11.5]$} & 71 & {$[18.5]$} & 68 & {$[8]$} \\
\hline Sex (male) & $52 \%$ & $(30)$ & $32 \%$ & (7) & $68 \%$ & $(15)$ & $57 \%$ & (8) \\
\hline Race (non-White) & $63 \%$ & $(36 / 57)$ & $55 \%$ & $(12)$ & $55 \%$ & 12 & $92 \%$ & $(12)$ \\
\hline BMI $\left(\mathrm{kg} / \mathrm{m}^{2}\right)$ & 27.6 & {$[7.9]$} & 26.1 & {$[5.3]$} & 28.2 & {$[10.2]$} & 29.5 & {$[9.9]$} \\
\hline Obesity (BMI > 30 kg/m²) & $37 \%$ & $(21 / 57)$ & $27 \%$ & (6) & $38 \%$ & $(8 / 21)$ & $50 \%$ & (7) \\
\hline \multicolumn{9}{|l|}{ Comorbidities } \\
\hline High cardiovascular risk profile ( $\geq 2$ of hypertension, hyperlipidemia, diabetes) & $55 \%$ & $(32)$ & $36 \%$ & (8) & $64 \%$ & $(14)$ & $71 \%$ & (10) \\
\hline Hypertension & $64 \%$ & $(37)$ & $59 \%$ & (13) & $59 \%$ & $(13)$ & $79 \%$ & $(11)$ \\
\hline Hyperlipidemia & $62 \%$ & (36) & $50 \%$ & $(11)$ & $59 \%$ & $(13)$ & $86 \%$ & $(12)$ \\
\hline Diabetes & $28 \%$ & $(16)$ & $9 \%$ & $(2)$ & $45 \%$ & $(10)$ & $29 \%$ & $(4)$ \\
\hline Previous atherosclerotic complications (CAD and/or CVA) & $22 \%$ & $(13)$ & $0 \%$ & $(0)$ & $36 \%$ & $(8)$ & $36 \%$ & (5) \\
\hline Congestive heart failure & $12 \%$ & (7) & $0 \%$ & $(0)$ & $14 \%$ & (3) & $29 \%$ & $(4)$ \\
\hline Current or former smoker & $37 \%$ & $(21 / 57)$ & $27 \%$ & (6) & $38 \%$ & $(8 / 21)$ & $50 \%$ & (7) \\
\hline Lung disease (COPD, emphysema, asthma, bronchiectasis) & $21 \%$ & $(12)$ & $18 \%$ & $(4)$ & $27 \%$ & $(6)$ & $14 \%$ & $(2)$ \\
\hline Chronic kidney disease (eGFR < 60 mL/min) & $24 \%$ & $(14)$ & $23 \%$ & (5) & $27 \%$ & $(6)$ & $21 \%$ & (3) \\
\hline History of other malignancy & $9 \%$ & (5) & $5 \%$ & $(1)$ & $5 \%$ & $(1)$ & $21 \%$ & (3) \\
\hline Number of comorbidities & 2 & {$[3]$} & 1 & {$[1.75]$} & 3 & {$[3]$} & 3.5 & {$[1.75]$} \\
\hline \multicolumn{9}{|l|}{ Concomitant medication } \\
\hline Anticoagulation & $21 \%$ & $(12)$ & $9 \%$ & $(2)$ & $27 \%$ & $(6)$ & $29 \%$ & (4) \\
\hline Aspirin & $59 \%$ & (34) & $59 \%$ & $(13)$ & $59 \%$ & $(13)$ & $57 \%$ & $(8)$ \\
\hline Statin & $47 \%$ & $(27)$ & $14 \%$ & (3) & $59 \%$ & $(13)$ & $79 \%$ & $(11)$ \\
\hline ACE inhibitor or angiotensin II receptor blocker & $45 \%$ & $(26)$ & $36 \%$ & (8) & $41 \%$ & (9) & $64 \%$ & (9) \\
\hline Beta blocker & $34 \%$ & $(20)$ & $9 \%$ & $(2)$ & $50 \%$ & $(11)$ & $50 \%$ & (7) \\
\hline Metformin & $16 \%$ & (9) & $5 \%$ & $(1)$ & $32 \%$ & (7) & $7 \%$ & $(1)$ \\
\hline Non-steroidal anti-inflammatory drug & $5 \%$ & (3) & $5 \%$ & $(1)$ & $5 \%$ & $(1)$ & $7 \%$ & $(1)$ \\
\hline Oral corticosteroids & $53 \%$ & $(31)$ & $45 \%$ & $(10)$ & $50 \%$ & $(11)$ & $71 \%$ & $(10)$ \\
\hline COVID-19 confirmed by PCR at MSH & $71 \%$ & $(41)$ & $59 \%$ & (13) & $86 \%$ & (19) & $64 \%$ & (9) \\
\hline
\end{tabular}

Note: values are presented as percentage $(n)$ or median [interquartile range]

Abbreviations: BMI body mass index, CAD coronary artery disease, CVA cerebrovascular accident, COPD chronic obstructive pulmonary disease, eGFR estimated glomerular filtration rate, ACE angiotensin-converting enzyme, NSAID non-steroidal anti-inflammatory drug, MSH Mount Sinai Hospital

support: 7 needed a nasal cannula or non-rebreather mask, 1 needed high flow oxygen, and 2 were immediately intubated and required mechanical ventilation.

During their hospital stay, 22 (95\%) patients developed fever, 18 (78\%) tachycardia (> $100 \mathrm{bpm})$, and $18(78 \%)$ hypoxemia $(\mathrm{SpO} 2<93 \%)$. Five $(22 \%)$ patients never required supplemental oxygen, and 10 (40\%) needed a nasal cannula or non-rebreather mask at some point during hospitalization. Four (17\%) patients were treated with high-flow oxygen, continuous positive airway pressure (CPAP) or bi-level positive airway pressure (BiPAP) machines and five (22\%) were eventually intubated. Seven $(30 \%)$ patients required intensive care unit (ICU) care during their hospitalization. The median length of stay was 22 days. Of the 23 patients admitted to our hospital with COVID-19, seven (30\%) died. When we consider the total hospitalized cohort (36 patients, i.e., including patients admitted at other hospitals), the mortality rate was $39 \%$ (14 patients). All 14 deaths were due to COVID-19. There were no deaths reported in patients who were not hospitalized among the total cohort.

Patients presented with multiple elevated inflammatory markers, including CRP (median: $89 \mathrm{mg} / \mathrm{L}$ ), ferritin (median: $595 \mu \mathrm{g} / \mathrm{L}$ ), IL-6 (median: $82.4 \mathrm{pg} / \mathrm{mL}$ ), whereas procalcitonin was normal (median: $0.2 \mathrm{ng} / \mathrm{mL}$ ). Leukocytes were not elevated (median: $4.3 \times 10^{9} / \mathrm{L}$ ) and the 
Table 2 Myeloma disease characteristics of patients

\begin{tabular}{|c|c|c|c|c|c|c|c|c|}
\hline \multirow{3}{*}{ Disease characteristics } & \multirow{2}{*}{\multicolumn{2}{|c|}{$\begin{array}{l}\text { All patients } \\
\boldsymbol{n}=58\end{array}$}} & \multirow{2}{*}{\multicolumn{2}{|c|}{$\begin{array}{l}\text { Patients not admitted to } \\
\text { hospital } \\
\boldsymbol{n}=22\end{array}$}} & \multirow{2}{*}{\multicolumn{2}{|c|}{$\begin{array}{l}\text { Hospitalized, discharged } \\
\text { alive } \\
\boldsymbol{n}=22\end{array}$}} & \multirow{2}{*}{\multicolumn{2}{|c|}{$\begin{array}{l}\text { Hospitalized, } \\
\text { deceased } \\
\boldsymbol{n}=14\end{array}$}} \\
\hline & & & & & & & & \\
\hline & \multirow{3}{*}{$7 \%$} & \multirow{3}{*}{ (4) } & \multirow{3}{*}{$9 \%$} & \multirow{3}{*}{ (2) } & \multirow{3}{*}{$5 \%$} & \multirow{3}{*}{$(1)$} & \multirow{3}{*}{$7 \%$} & \multirow{3}{*}{$(1)$} \\
\hline SMM & & & & & & & & \\
\hline SMM/MM subtype & & & & & & & & \\
\hline $\lg D$ & $2 \%$ & $(1)$ & $5 \%$ & (1) & $0 \%$ & $(0)$ & $0 \%$ & $(0)$ \\
\hline $\lg G$ & $59 \%$ & (34) & $59 \%$ & (13) & $64 \%$ & $(14)$ & $50 \%$ & (7) \\
\hline $\lg A$ & $19 \%$ & $(11)$ & $23 \%$ & (5) & $14 \%$ & (3) & $21 \%$ & (3) \\
\hline Light chain disease & $33 \%$ & (19) & $23 \%$ & (5) & $41 \%$ & (9) & $36 \%$ & (5) \\
\hline Extramedullary disease history & $31 \%$ & $(18)$ & $36 \%$ & (8) & $32 \%$ & (7) & $21 \%$ & (3) \\
\hline \multicolumn{9}{|l|}{ ISS at diagnosis } \\
\hline 1 & $33 \%$ & $(18 / 54)$ & $45 \%$ & $(9 / 20)$ & $43 \%$ & $(9 / 21)$ & $0 \%$ & $(0 / 13)$ \\
\hline 2 & $26 \%$ & $(14 / 54)$ & $25 \%$ & $(5 / 20)$ & $33 \%$ & $(7 / 21)$ & $15 \%$ & $(2 / 13)$ \\
\hline 3 & $19 \%$ & $(10 / 54)$ & $20 \%$ & $(4 / 20)$ & $14 \%$ & $(3 / 21)$ & $23 \%$ & $(3 / 13)$ \\
\hline Not known & $22 \%$ & $(12 / 54)$ & $10 \%$ & $(2 / 20)$ & $10 \%$ & $(2 / 21)$ & $62 \%$ & $(8 / 13)$ \\
\hline High-risk cytogenetics & $39 \%$ & $(22 / 56)$ & $33 \%$ & $(7 / 21)$ & $41 \%$ & (9) & $46 \%$ & $(6 / 13)$ \\
\hline Time since MM diagnosis (months) & 29.8 & {$[44.2]$} & 44.8 & [38.7] & 27.2 & {$[55.8]$} & 28.6 & {$[23.6]$} \\
\hline History of ASCT & $41 \%$ & $(22 / 54)$ & $60 \%$ & $(12 / 20)$ & $24 \%$ & $(5 / 21)$ & $38 \%$ & $(5 / 13)$ \\
\hline Time since ASCT (days) & 985 & [1590] & 985 & [744] & 2148 & {$[2206]$} & 460 & [1734] \\
\hline ASCT within last year & $9 \%$ & $(5 / 54)$ & $15 \%$ & $(3 / 20)$ & $0 \%$ & $(0 / 21)$ & $15 \%$ & $(2 / 13)$ \\
\hline Lines of therapy $(n)$ & 1.5 & {$[2]$} & 2 & {$[2]$} & 2 & {$[3]$} & 1 & {$[1]$} \\
\hline More than 4 lines of treatment & $17 \%$ & $(9 / 54)$ & $20 \%$ & $(4 / 20)$ & $19 \%$ & $(4 / 21)$ & $8 \%$ & $(1 / 13)$ \\
\hline ECOG 0 & $47 \%$ & $(27)$ & $64 \%$ & (14) & $41 \%$ & (9) & $29 \%$ & (4) \\
\hline \multicolumn{9}{|l|}{ Current response status* } \\
\hline$s C R$ or $C R$ & $26 \%$ & (15) & $45 \%$ & (10) & $14 \%$ & (3) & $14 \%$ & $(2)$ \\
\hline VGPR & $19 \%$ & $(11)$ & $18 \%$ & (4) & $14 \%$ & (3) & $29 \%$ & (4) \\
\hline$P R$ & $22 \%$ & (13) & $18 \%$ & (4) & $23 \%$ & (5) & $29 \%$ & (4) \\
\hline SD & $3 \%$ & (2) & $5 \%$ & (1) & $5 \%$ & $(1)$ & $0 \%$ & $(0)$ \\
\hline PD & $16 \%$ & (9) & $5 \%$ & (1) & $23 \%$ & (5) & $21 \%$ & (3) \\
\hline Not evaluable & $14 \%$ & (8) & $9 \%$ & (2) & $23 \%$ & (5) & $7 \%$ & $(1)$ \\
\hline \multicolumn{9}{|l|}{ Current MM treatment regimen } \\
\hline Contains CD38 mAb & $48 \%$ & $(28)$ & $50 \%$ & (11) & $50 \%$ & $(11)$ & $43 \%$ & (6) \\
\hline Contains IMiD & $55 \%$ & $(32)$ & $55 \%$ & (12) & $59 \%$ & $(13)$ & $50 \%$ & (7) \\
\hline Contains proteasome inhibitor & $38 \%$ & $(22)$ & $27 \%$ & (6) & $41 \%$ & (9) & $50 \%$ & (7) \\
\hline Contains corticosteroids & $52 \%$ & $(30)$ & $45 \%$ & (10) & $50 \%$ & $(11)$ & $64 \%$ & $(9)$ \\
\hline Contains venetoclax & $9 \%$ & (5) & $14 \%$ & (3) & $9 \%$ & $(2)$ & $0 \%$ & $(0)$ \\
\hline No active treatment & $19 \%$ & $(11)$ & $18 \%$ & (4) & $18 \%$ & $(4)$ & $21 \%$ & (3) \\
\hline \multicolumn{9}{|c|}{ Biochemical parameters at last clinic visit before COVID-19 episode } \\
\hline Leukocyte count $(\times 10 \mathrm{e} / \mathrm{L})$ & 4.6 & {$[2.1]$} & 4.1 & {$[2.1]$} & 5.2 & {$[2.4]$} & 4.7 & {$[1.5]$} \\
\hline Leukocytopenia $(<4 \times 10 \mathrm{e} 9 / \mathrm{L})$ & $35 \%$ & $(20 / 57)$ & $41 \%$ & (9) & $33 \%$ & $(7 / 21)$ & $29 \%$ & (4) \\
\hline Absolute neutrophil count (× 10e9/L) & 2.6 & {$[2.2]$} & 2.4 & [1] & 3.4 & {$[3]$} & 2.4 & {$[1.4]$} \\
\hline Neutropenia $(<2 \times 10 \mathrm{e} / \mathrm{L})$ & $26 \%$ & $(15 / 57)$ & $32 \%$ & (7) & $29 \%$ & $(6 / 21)$ & $14 \%$ & $(2)$ \\
\hline Absolute lymphocyte count (× 10e9/L) & 1 & {$[0.8]$} & 1.1 & {$[1.2]$} & 0.8 & {$[0.9]$} & 1.2 & {$[0.5]$} \\
\hline Lymphocytopenia (< 0.5 × 10e9/L) & $12 \%$ & $(7 / 57)$ & $0 \%$ & $(0)$ & $33 \%$ & $(7 / 21)$ & $0 \%$ & $(0)$ \\
\hline
\end{tabular}


Table 2 Myeloma disease characteristics of patients (Continued)

\begin{tabular}{|c|c|c|c|c|c|c|c|c|}
\hline \multirow[b]{3}{*}{ Serum free light chain ratio (involved/uninvolved) } & \multirow{2}{*}{\multicolumn{2}{|c|}{$\begin{array}{l}\text { All patients } \\
\boldsymbol{n}=58\end{array}$}} & \multirow{2}{*}{\multicolumn{2}{|c|}{$\begin{array}{l}\text { Patients not admitted to } \\
\text { hospital } \\
\boldsymbol{n}=22\end{array}$}} & \multirow{2}{*}{\multicolumn{2}{|c|}{$\begin{array}{l}\text { Hospitalized, discharged } \\
\text { alive } \\
\boldsymbol{n}=22\end{array}$}} & \multirow{2}{*}{\multicolumn{2}{|c|}{$\begin{array}{l}\text { Hospitalized, } \\
\text { deceased } \\
\boldsymbol{n}=14\end{array}$}} \\
\hline & & & & & & & & \\
\hline & 2.5 & [11.9] & 1.6 & {$[3.3]$} & 7.7 & {$[45.5]$} & 1.8 & {$[5.2]$} \\
\hline M spike $(\mathrm{g} / \mathrm{dL})$ & 0 & {$[0.6]$} & 0 & {$[0.2]$} & 0.3 & {$[1.2]$} & 0.1 & {$[0.4]$} \\
\hline $\operatorname{lgG}(\mathrm{mg} / \mathrm{dL})$ & 805 & [736] & 1074.5 & {$[683.8]$} & 764 & [1121] & 727 & [496.8] \\
\hline Hypogammaglobulinemia (lgG < 700 mg/dL) & $37 \%$ & $(21 / 57)$ & $32 \%$ & (7) & $38 \%$ & $(8 / 21)$ & $43 \%$ & $(6)$ \\
\hline Severe hypogammaglobulinemia (lgG < 400 mg/dL) & $11 \%$ & $(6 / 57)$ & $0 \%$ & (0) & $10 \%$ & $(2 / 21)$ & $29 \%$ & (4) \\
\hline Immunoparesis & $89 \%$ & $(51 / 57)$ & $82 \%$ & (18) & $95 \%$ & $(20 / 21)$ & $93 \%$ & (13) \\
\hline
\end{tabular}

Note: values are presented as percentage $(n)$ or median [interquartile range]

Abbreviations: SMM smoldering multiple myeloma, Ig immunoglobulin, ISS international staging system, MM multiple myeloma, $A S C T$ autologous stem cell transplant, ECOG Eastern Cooperative Oncology Group, $s C R$ stringent complete response, $C R$ complete response, VGPR very good partial response, $S D$ stable disease, $P D$ progressive disease, $m A b$ monoclonal antibody, IMiD immunomodulatory agent

*According to IMWG criteria

absolute lymphocyte count was low (median $0.6 \times 10^{9} /$ L) whereas absolute neutrophil count was within the normal range (median $3.6 \times 10^{9} / \mathrm{L}$ ). On initial presentation, lactate dehydrogenase (LDH, median $249.5 \mathrm{U} / \mathrm{L}$ ), fibrinogen (median $600 \mathrm{mg} / \mathrm{dL}$ ), and D-dimer (median 1.2 $\mathrm{mg} / \mathrm{L})$ were elevated but transaminases were normal (median aspartate aminotransferase (AST) and alanine aminotransferase (ALT): $24 \mathrm{U} / \mathrm{L}$ and $20 \mathrm{U} / \mathrm{L}$, respectively). Peak levels for these markers are shown in Table 3 and temporal trends for a subset of patients are illustrated in Supplementary Figure S1. CRP and ferritin peaked early (within the first 10 days of hospitalization) and subsequently demonstrated a downward trend over time, with a slower decline in ferritin levels notable in patients that eventually died. D-dimer level was transiently elevated in some patients but was persistently and progressively elevated in all patients that eventually died. The inflammatory cytokines IL- $1 \beta$, IL- 6 , IL-8, and TNF$\alpha$ were assessed over the duration of hospitalization at the discretion of the treating physician. Peak levels for IL-6, IL-8, and TNF- $\alpha$ were elevated (median: $128.5 \mathrm{pg} /$ $\mathrm{mL}, \quad 65.4 \mathrm{pg} / \mathrm{mL}$, and $28.7 \mathrm{pg} / \mathrm{mL}$, respectively), and levels for IL-1 $\beta$ were generally low (median: $0.5 \mathrm{pg} / \mathrm{mL}$ ).

We describe COVID-19 management comprehensively for the 23 patients hospitalized at our center and their outcome (Table 3). One (4\%) patient received remdesivir, 17 (74\%) patients received hydroxychloroquine, and 17 (74\%) patients received azithromycin. Nineteen (82\%) patients were treated with other antibiotics, most commonly a beta-lactam antibiotic \pm vancomycin $(n=16)$ for presumed bacterial superinfection. Six (26\%) patients received granulocyte colony-stimulating factor (G-CSF) and $18(78 \%)$ patients received therapeutic anticoagulation, 13 of which had not been on full anticoagulation before COVID-19. Patients were treated with a direct oral anticoagulant (DOAC, $n=3$ ), therapeutic doses of enoxaparin $(n=8)$, or a heparin drip $(n=2)$. There were no major bleeding events. Ten (43\%) patients were given systemic corticosteroids. One (4\%) patient was treated with convalescent plasma. Anti-TNF $\alpha$, anti-IL-6, and anti-IL-1 therapy were initiated in 1 (4\%), 4 (17\%), and 2 (9\%) patients, respectively. Five patients $(22 \%)$ were given low-dose selinexor, a selective inhibitor of nuclear export, for its presumed activity against virus host protein interaction [22] and to counter amplification of proinflammatory signaling [23].

\section{Antibody serology and repeat PCR testing}

We collected data on antibody testing and follow-up PCR testing for patients. As of May 28, 2020, 96\% (22/ 23) of patients have developed antibodies against SARS$\mathrm{CoV}-2$ at a median time of 32 (range 6-50) days since COVID-19 diagnosis. Titers ranged from 1:160 to 1 : 2880 , with $74 \%(17 / 23)$ exhibiting the most significant titer level of $1: 2880,9 \%(2 / 23)$ with $1: 960,9 \%(2 / 23)$ with $1: 320$, and $4 \%(1 / 23)$ with $1: 160$. Antibody titer did not correlate with COVID-19 severity, yet we observed that all 5 MM patients with low titers $(<1: 2880)$ had hypogammaglobulinemia. The one patient who did not develop any antibodies had SMM and was tested 27 days after initial diagnosis. So far, 27 patients have undergone repeat PCR testing; 74\% (20/27) are negative and the median time to PCR negativity was 43 (range 19-68) days from initial positive PCR. Among the 22 patients with positive antibody titers, 19 patients had repeat PCR swab and 3 remained positive while 16 were negative.

\section{Clinical associations}

In a univariate analysis on all patients, we found that the following variables were significantly associated with hospitalization, as shown in Table 4: age over 70 (OR 7.74, $p=0.007$ ), male sex (OR 3.70, $p=0.030$ ), diabetes mellitus type 2 (OR 6.18, $p=0.016$ ), high cardiovascular risk profile (OR 3.42, $p=0.032$ ), history of CAD (OR $\infty$, $p=0.009)$, history of CHF (OR $\infty, p=0.037)$, use of statins $(\mathrm{OR}=12.10, p<0.001)$ and use of beta-blockers 
Table 3 Clinical parameters, treatments, and outcomes of patients hospitalized due to COVID-19 at Mount Sinai Hospital

\begin{tabular}{|c|c|c|c|c|c|c|}
\hline \multirow{2}{*}{$\begin{array}{l}\text { Subset of patients treated at Mount Sinai hospital } \\
\text { for which full clinical data was available }\end{array}$} & \multicolumn{2}{|c|}{ Hospitalized patients total } & \multicolumn{2}{|c|}{ Hospitalized patients alive } & \multicolumn{2}{|c|}{ Hospitalized patients deceased } \\
\hline & \multicolumn{2}{|l|}{$\boldsymbol{n}=23$} & \multicolumn{2}{|c|}{$\boldsymbol{n}=16$} & \multicolumn{2}{|l|}{$\boldsymbol{n}=7$} \\
\hline \multicolumn{7}{|l|}{ Clinical presentation } \\
\hline Fever & $70 \%$ & $(16)$ & $69 \%$ & (11) & $71 \%$ & (5) \\
\hline Systolic BP $<90$ mmHg & $9 \%$ & $(2)$ & $0 \%$ & (0) & $29 \%$ & (2) \\
\hline MAP $<65 \mathrm{mmHg}$ & $9 \%$ & $(2)$ & $0 \%$ & (0) & $29 \%$ & (2) \\
\hline Heart rate $>100 \mathrm{bpm}$ & $48 \%$ & $(11)$ & $56 \%$ & (9) & $29 \%$ & (2) \\
\hline $\mathrm{RR}>20 / \mathrm{min}$ & $26 \%$ & (6) & $19 \%$ & (3) & $43 \%$ & (3) \\
\hline \multicolumn{7}{|l|}{ Oxygen requirement at presentation } \\
\hline None & $57 \%$ & (13) & $69 \%$ & (11) & $29 \%$ & (2) \\
\hline Nasal cannula or NRB mask & $30 \%$ & (7) & $25 \%$ & (4) & $43 \%$ & (3) \\
\hline High-flow oxygen, CPAP, or BiPAP & $4 \%$ & $(1)$ & $0 \%$ & (0) & $14 \%$ & (1) \\
\hline Mechanical ventilation & $9 \%$ & (2) & $6 \%$ & (1) & $14 \%$ & (1) \\
\hline \multicolumn{7}{|l|}{ Treatment initiated } \\
\hline Remdesivir & $4 \%$ & (1) & $6 \%$ & (1) & $0 \%$ & (0) \\
\hline Hydroxychloroquine & $74 \%$ & $(17)$ & $69 \%$ & (11) & $86 \%$ & (6) \\
\hline Azithromycin & $74 \%$ & $(17)$ & $75 \%$ & (12) & $71 \%$ & (5) \\
\hline Other antibiotics & $83 \%$ & (19) & $81 \%$ & (13) & $86 \%$ & (6) \\
\hline G-CSF & $26 \%$ & (6) & $38 \%$ & (6) & $0 \%$ & (0) \\
\hline Therapeutic anticoagulation & $78 \%$ & $(18)$ & $69 \%$ & (11) & $100 \%$ & (7) \\
\hline Systemic corticosteroids & $43 \%$ & $(10)$ & $31 \%$ & (5) & $71 \%$ & (5) \\
\hline Anti-TNF & $4 \%$ & $(1)$ & $0 \%$ & (0) & $14 \%$ & (1) \\
\hline Anti-IL-1 & $9 \%$ & $(2)$ & $0 \%$ & (0) & $29 \%$ & (2) \\
\hline Anti-IL-6 & $17 \%$ & (4) & $13 \%$ & (2) & $29 \%$ & (2) \\
\hline Selinexor & $22 \%$ & (5) & $25 \%$ & (4) & $14 \%$ & (1) \\
\hline Convalescent plasma & $4 \%$ & (1) & $6 \%$ & (1) & $0 \%$ & (0) \\
\hline
\end{tabular}

\section{Complications}

Highest level of oxygen requirement

None
Nasal cannula or NRB mask
High-flow oxygen, CPAP, or BiPAP
Mechanical ventilation
ICU
Acute kidney injury
Shock
Sepsis or HAPNAP
C. difficile infection
Cardiac complication

Worst biochemical parameters

CRP
Total leukocyte count $(\times 10 \mathrm{e} / \mathrm{L})$ (lowest)
Absolute lymphocyte count $(\times 10 \mathrm{e} 9 / \mathrm{L})$ (lowest)
Creatinine $(\mathrm{mg} / \mathrm{dL})$
Procalcitonin $(\mathrm{ng} / \mathrm{mL})$
Ferritin $(\mu \mathrm{g} / \mathrm{L})$

$\begin{array}{llllll}26 \% & (6) & 31 \% & (5) & 14 \% & (1) \\ 39 \% & (9) & 56 \% & (9) & 0 \% & (0) \\ 13 \% & (3) & 6 \% & (1) & 29 \% & (2) \\ 22 \% & (5) & 6 \% & (1) & 57 \% & (4) \\ 30 \% & (7) & 6 \% & (1) & 86 \% & (6) \\ 52 \% & (12) & 38 \% & (6) & 86 \% & (6) \\ 30 \% & (7) & 0 \% & (0) & 100 \% & (7) \\ 9 \% & (2) & 6 \% & (1) & 14 \% & (1) \\ 4 \% & (1) & 6 \% & (1) & 0 \% & (0) \\ 30 \% & (7) & 19 \% & (3) & 57 \% & (4) \\ & & & & & {[132.9]} \\ 151.5 & {[178.1]} & 144.9 & {[107.7]} & 294.1 & {[8.4]} \\ 3.3 & {[2.6]} & 2.9 & {[2.4]} & 4.3 & {[0.2]} \\ 0.3 & {[0.4]} & 0.4 & {[0.5]} & 0.2 & {[2]} \\ 1.6 & {[2.4]} & 1.1 & {[1.4]} & 2.7 & {[9.9]} \\ 0.8 & {[2.1]} & 0.5 & {[0.8]} & 2.7 & {[2018]} \\ 2537 & {[2578]} & 1282 & {[2170.5]} & 3409 & \end{array}$


Table 3 Clinical parameters, treatments, and outcomes of patients hospitalized due to COVID-19 at Mount Sinai Hospital (Continued)

\begin{tabular}{|c|c|c|c|c|c|c|}
\hline \multirow{2}{*}{$\begin{array}{l}\text { Subset of patients treated at Mount Sinai hospital } \\
\text { for which full clinical data was available }\end{array}$} & \multicolumn{2}{|c|}{ Hospitalized patients total } & \multicolumn{2}{|c|}{ Hospitalized patients alive } & \multicolumn{2}{|c|}{ Hospitalized patients deceased } \\
\hline & $\boldsymbol{n}=23$ & & $\boldsymbol{n}=16$ & & $\boldsymbol{n}=7$ & \\
\hline Fibrinogen (mg/dL) & 667 & [167] & 646 & [209.5] & 673.5 & {$[43.8]$} \\
\hline D-dimer (mg/L) & 2.5 & {$[14.3]$} & 2 & {$[2.6]$} & 20 & {$[7.6]$} \\
\hline $\mathrm{LDH}(\mathrm{U} / \mathrm{L})$ & 531.5 & {$[515.8]$} & 478 & [329] & 739 & [344] \\
\hline $\mathrm{ALT}(\mathrm{U} / \mathrm{L})$ & 61 & {$[58.5]$} & 43.5 & {$[52.8]$} & 79 & {$[32]$} \\
\hline AST (U/L) & 78 & {$[62.5]$} & 49.5 & {$[54.3]$} & 101 & {$[51.5]$} \\
\hline $\mathrm{IL}-1 \beta(\mathrm{pg} / \mathrm{mL})$ & 0.5 & {$[0.9]$} & 0.5 & {$[0.8]$} & 0.5 & {$[0.9]$} \\
\hline IL-6 (pg/mL) & 128.5 & {$[211.6]$} & 119.3 & {$[102.4]$} & 296.8 & {$[821.2]$} \\
\hline IL-8 (pg/mL) & 65.4 & {$[62.3]$} & 46.6 & {$[67.6]$} & 137 & {$[89.6]$} \\
\hline TNF-alfa (pg/mL) & 28.7 & [11] & 29.4 & {$[8.4]$} & 21.3 & {$[15.2]$} \\
\hline
\end{tabular}

Note: values are presented as percentage $(n)$ or median [interquartile range]

Abbreviations: $B P$ blood pressure, $M A P$ mean arterial pressure, $\mathrm{SpO}_{2}$ oxygen saturation, $R R$ respiratory rate, NRB non-rebreather, $C P A P$ continuous positive airway pressure, BiPAP bi-level positive airway pressure, G-CSF granulocyte colony stimulating factor, TNF tumor necrosis factor, IL interleukin, ICU intensive care unit, HAP hospital-acquired pneumonia, VAP ventilator-associated pneumonia, CRP C-reactive protein, LDH lactate dehydrogenase, ALT alanine aminotransferase, AST aspartate aminotransferase

(OR 9.63, $p=0.002)$. We also noted significant associations between hospitalization status and grade 3 lymphocytopenia (OR $\infty, p=0.036$ ) at the last clinic visit prior to COVID-19 infection. Patients who had not achieved a $\mathrm{CR}$ or $\mathrm{sCR}$ were at increased risk of hospitalization $(p=$ 0.013).

Similarly, for hospitalized patients, using a univariate approach, we found a statistically significant association between mortality and these variables: non-White race (OR 10.49, $p=0.011$ ), statin use (OR 6.21, $p=0.012$ ), severe hypogammaglobulinemia (OR 7.80, $p=0.027$ ), and higher peak levels of D-dimer $(p=0.004)$, ferritin $(p$ $=0.007)$, procalcitonin $(p=0.010)$, and CRP $(p=0.019)$. The full list of associations is shown in Table 4 .

\section{Discussion}

Situated in the heart of New York City, our cancer center at Mount Sinai Hospital bore witness to the immense disruption of healthcare services caused by COVID-19. During the initial phase of the pandemic, the goal was to keep patients at home following federal and state guidelines of isolation, social distancing, and strict hand hygiene [2426]. Patients were switched to all oral regimens if possible or had delayed therapy depending on the perceived risk of need for therapy to control myeloma versus exposure to SARS-CoV-2. Yet community transmission of SARS-CoV2 during the pandemic was inevitable.

There were no deaths among myeloma patients with milder symptoms who were managed entirely as outpatients with COVID-19 in this cohort. The mortality rates of the overall cohort $(n=58)$, MM patients admitted to Mount Sinai Hospital $(n=23)$, and all admitted MM patients $(n=36)$ were $24 \%, 30 \%$, and $39 \%$, respectively. These figures are in line with the overall mortality seen in New York, where the estimated mortality among hospitalized patients over 45 years old is $37 \%$ as of May 25, 2020 [1, 27]. Interestingly, the mortality among our cohort of MM patients was lower than the $54.6 \%$ seen in a cohort of $75 \mathrm{MM}$ patients treated in Britain [28]. We acknowledge that the apparent mortality differences between different countries and health systems may be affected by the local epidemiology, hospitalization, and resource utilization rates and potential differences in the escalation of care. However, in both of these populations, there appeared to be a trend toward increased mortality in patients of non-White/Caucasian background. This has been consistently seen in the USA, where death rates for COVID-19 are several fold higher in patients of Black and Hispanic origins [29-31].

MM specific disease characteristics and the type of MM treatment were not associated with increased mortality. In contrast, we observed that age and cardiovascular risk factors (diabetes, CAD, CHF) were significantly associated with patient hospitalization for COVID-19. The data from our cohort showed that non-White background, severe (< $400 \mathrm{mg} / \mathrm{dL}$ ) hypogammaglobulinemia, and statin use were significantly associated with mortality. This information would indicate that during the post pandemic phase, we do not have to change the management of myeloma patients. However, earlier diagnosis of COVID-19 and prompt intervention especially for the vulnerable population identified above is warranted to reduce the risk of mortality. As we reopen and move forward into a post-COVID-19 era, we will need to remain vigilant, particularly for select patient groups, and await effective COVID-19 treatments while balancing the need to manage patients' myeloma.

We were able to capture the evolution of inflammatory markers for patients who were admitted to the inpatient service, and we found a significant association with 
Table 4 Univariate associations of selected variables with risk of hospitalization and mortality

\begin{tabular}{|c|c|c|c|c|}
\hline & $\begin{array}{l}\text { Hospitalized vs } \\
\text { non-hospitalized }\end{array}$ & $n=58$ & Mortality (dead vs alive) & $n=58$ \\
\hline & OR $[95 \% \mathrm{Cl}$ ] or median $\mathrm{NH} / \mathrm{H}$ & $\boldsymbol{p}$ value & OR [95\% Cl] or median dead/alive & $\begin{array}{l}p \\
\text { value }\end{array}$ \\
\hline \multicolumn{5}{|l|}{ Demographics } \\
\hline Age (> 70 years) & $7.74[1.51-78.12]$ & 0.007 & $1.32[0.29-5.47]$ & 0.744 \\
\hline Sex (male) & $3.7[1.08-13.81]$ & 0.030 & $1.33[0.34-5.48]$ & 0.762 \\
\hline Race (non-White) & $1.87[0.55-6.51]$ & 0.275 & $10.49[1.35-481.76]$ & 0.011 \\
\hline Obesity (BMI $\geq 30$ kg/m²) & $1.98[0.56-7.72]$ & 0.272 & $2.04[0.5-8.4]$ & 0.340 \\
\hline \multicolumn{5}{|l|}{ Comorbidities } \\
\hline $\begin{array}{l}\text { High cardiovascular risk profile } \\
\text { ( } \geq 2 \text { of hypertension, hyperlipidemia, diabetes) }\end{array}$ & $3.42[1.01-12.4]$ & 0.032 & $2.46[0.59-12.43]$ & 0.221 \\
\hline Hypertension & $1.38[0.4-4.73]$ & 0.585 & $2.5[0.55-15.93]$ & 0.220 \\
\hline Hyperlipidemia & $2.24[0.66-7.81]$ & 0.170 & $4.88[0.92-49.98]$ & 0.057 \\
\hline Diabetes & $6.18[1.19-62.84]$ & 0.016 & $1.07[0.2-4.67]$ & 1.000 \\
\hline Coronary artery disease & $\operatorname{lnf}[1.64-\operatorname{Inf}]$ & 0.009 & $2.49[0.43-13.07]$ & 0.233 \\
\hline Stroke & $\infty[0.58-\infty]$ & 0.145 & $2.24[0.17-22.05]$ & 0.585 \\
\hline Congestive heart failure & $\infty[0.96-\infty]$ & 0.037 & $5.26[0.76-41.93]$ & 0.051 \\
\hline Current or former smoker & $1.98[0.56-7.72]$ & 0.272 & $2.04[0.5-8.4]$ & 0.340 \\
\hline Lung disease (COPD, emphysema, asthma, bronchiectasis) & $1.28[0.29-6.69]$ & 1.000 & $0.57[0.05-3.3]$ & 0.711 \\
\hline Chronic kidney disease (eGFR < 60 mL/min) & $1.13[0.28-5.06]$ & 1.000 & $0.82[0.12-3.97]$ & 1.000 \\
\hline History of other malignancy & $2.59[0.23-135.24]$ & 0.640 & $5.51[0.56-73.43]$ & 0.085 \\
\hline Number of comorbidities & $1 / 3$ & 0.011 & $2 / 3.5$ & 0.055 \\
\hline \multicolumn{5}{|l|}{ Concomitant medications } \\
\hline Anticoagulation & $3.77[0.69-39.19]$ & 0.108 & $1.78[0.32-8.51]$ & 0.457 \\
\hline ACE inhibitor or angiotensin II receptor blocker & $1.73[0.52-6.06]$ & 0.416 & $2.81[0.7-12.6]$ & 0.126 \\
\hline Beta blocker & $9.63[1.89-97.14]$ & 0.002 & $2.35[0.58-9.72]$ & 0.203 \\
\hline Metformin & $5.85[0.69-278.29]$ & 0.133 & $0.35[0.01-3.08]$ & 0.431 \\
\hline Statin & $12.06[2.78-76.13]$ & $<0.001$ & $6.21[1.37-39.77]$ & 0.012 \\
\hline Aspirin & $0.97[0.28-3.23]$ & 1.000 & $0.92[0.23-3.83]$ & 1.000 \\
\hline NSAID & $1.23[0.06-76.27]$ & 1.000 & $1.6[0.03-33.14]$ & 1.000 \\
\hline Oral corticosteroids & $1.66[0.51-5.61]$ & 0.420 & $2.69[0.65-13.59]$ & 0.139 \\
\hline \multicolumn{5}{|l|}{ Disease characteristics } \\
\hline Light chain disease & $2.09[0.44-13.55]$ & 0.342 & $1.19[0.26-4.88]$ & 1.000 \\
\hline High risk cytogenetics & $1.49[0.43-5.52]$ & 0.577 & $1.44[0.33-6.03]$ & 0.747 \\
\hline \multicolumn{5}{|l|}{ Current response status } \\
\hline$s C R$ or $C R$ & $0.2[0.04-0.8]$ & 0.013 & $0.4[0.04-2.23]$ & 0.317 \\
\hline \multicolumn{5}{|l|}{ Current $\mathrm{MM}$ treatment regimen } \\
\hline Contains CD38 mAb & $0.9[0.27-2.95]$ & 1.000 & $0.75[0.18-2.96]$ & 0.762 \\
\hline Contains IMiD & $1.04[0.31-3.43]$ & 1.000 & $0.76[0.19-3.04]$ & 0.761 \\
\hline Contains proteasome inhibitor & $2.11[0.6-8.17]$ & 0.267 & $1.91[0.47-7.78]$ & 0.350 \\
\hline Contains corticosteroids & $1.49[0.45-4.99]$ & 0.589 & $1.95[0.49-8.67]$ & 0.363 \\
\hline Contains venetoclax & $0.38[0.03-3.62]$ & 0.357 & $0[0-3.45]$ & 0.322 \\
\hline No active treatment & $1.08[0.23-5.79]$ & 1.000 & $1.22[0.18-6.34]$ & 1.000 \\
\hline \multicolumn{5}{|c|}{ Biochemical parameters at last clinic visit before COVID-19 episode } \\
\hline Leukocytopenia $(<4 \times 10 \mathrm{e} / \mathrm{L})$ & $0.67[0.19-2.34]$ & 0.571 & $0.68[0.13-2.88]$ & 0.749 \\
\hline
\end{tabular}


Table 4 Univariate associations of selected variables with risk of hospitalization and mortality (Continued)

\begin{tabular}{|c|c|c|c|c|}
\hline & $\begin{array}{l}\text { Hospitalized vs } \\
\text { non-hospitalized }\end{array}$ & $n=58$ & Mortality (dead vs alive) & \multirow{2}{*}{$\begin{array}{l}n=58 \\
\boldsymbol{p} \\
\text { value }\end{array}$} \\
\hline & $\mathrm{OR}[95 \% \mathrm{Cl}]$ or median $\mathrm{NH} / \mathrm{H}$ & $\boldsymbol{p}$ value & OR $[95 \% \mathrm{Cl}]$ or median dead/alive & \\
\hline Lymphocytopenia $(<0.5 \times 10 \mathrm{e} 9 / \mathrm{L})$ & $\infty[0.99-\infty]$ & 0.036 & $0[0-2.07]$ & 0.176 \\
\hline Neutropenia $(<2 \times 10 \mathrm{e} 9 / \mathrm{L})$ & $0.64[0.16-2.52]$ & 0.542 & $0.39[0.04-2.16]$ & 0.312 \\
\hline lgG level (mg/dL) & $1074.5 / 738$ & 0.297 & $869 / 718$ & 0.074 \\
\hline Hypogammaglobulinemia (lgG < 700 mg/dL) & $1.42[0.41-5.24]$ & 0.584 & $1.39[0.33-5.61]$ & 0.751 \\
\hline Severe hypogammaglobulinemia (lgG < 400 mg/dL) & $\infty[0.79-\infty]$ & 0.072 & $7.80[0.97-97.75]$ & 0.027 \\
\hline Immunoparesis & $3.58[0.46-43.2]$ & 0.192 & $1.70[0.17-87.01]$ & 1.000 \\
\hline Peak biochemical parameters during hospitalization & \multicolumn{3}{|c|}{$\begin{array}{l}\text { Note: variables below only apply to the subset of patients hospitalized at Mount } \\
\text { Sinai Hospital }\end{array}$} & $n=23$ \\
\hline CRP $(\mathrm{mg} / \mathrm{L})$ & - & - & $144.8 / 289.4$ & 0.019 \\
\hline Total leukocyte count (× 10e9/L) (lowest) & - & - & $2.9 / 4.2$ & 0.444 \\
\hline Absolute lymphocyte count (× 10e9/L) (lowest) & - & - & $0.4 / 0.2$ & 0.319 \\
\hline Creatinine (mg/dL) & - & - & $1.1 / 2.5$ & 0.052 \\
\hline Procalcitonin (ng/mL) & - & - & $0.5 / 2.3$ & 0.010 \\
\hline Ferritin $(\mu \mathrm{g} / \mathrm{L})$ & - & - & $1282 / 3474$ & 0.007 \\
\hline Fibrinogen (mg/dL) & - & - & $646 / 667$ & 0.888 \\
\hline D-dimer (mg/L) & - & - & $2 / 18.2$ & 0.004 \\
\hline $\mathrm{LDH}(\mathrm{U} / \mathrm{L})$ & - & - & $478 / 830$ & 0.065 \\
\hline $\mathrm{ALT}(\mathrm{U} / \mathrm{L})$ & - & - & $43.5 / 76.5$ & 0.244 \\
\hline AST (U/L) & - & - & $49.5 / 100$ & 0.054 \\
\hline IL-1b (pg/mL) & - & - & $0.5 / 0.5$ & 1.000 \\
\hline IL-6 (pg/mL) & - & - & $119.2 / 296.8$ & 0.117 \\
\hline IL-8 (pg/mL) & - & - & $46.6 / 137$ & 0.104 \\
\hline TNF-alfa (pg/mL) & - & - & $29.4 / 21.3$ & 0.574 \\
\hline
\end{tabular}

Note: values are presented as OR [95\% confidence interval] or median of group 1/median of group 2; $p$ values according to Wilcoxon test for continuous variables and Fisher's exact test for categorical variables

Abbreviations: $O R$ odds ratio, $C l$ confidence interval, $N H$ not hospitalized, $H$ hospitalized, BMI body mass index, COPD chronic obstructive pulmonary disease, eGFR estimated glomerular filtration rate, $A C E$ angiotensin-converting enzyme, NSAID non-steroidal anti-inflammatory drug, ASCT autologous stem cell transplant, $1 g$ immunoglobulin, CRP C-reactive protein, ALT alanine aminotransferase, AST aspartate aminotransferase, IL interleukin, TNF tumor necrosis factor

mortality in patients who had elevated D-dimer, CRP, or ferritin. Many COVID-19 patients treated at our institution also received a rapid panel for cytokine testing as part of a larger study to characterize the inflammatory profile of COVID-19 illness. Among our cohort of hospitalized patients, those who died appeared to exhibit rather elevated pro-inflammatory cytokines, consistent in principle with what was seen in a large COVID-19 cohort analyzed at the Mount Sinai Health System [32]. It is possible that a CRS-like syndrome, similar though not identical to one seen in MM patients treated with CAR$\mathrm{T}$ cells [13, 33] and bispecific antibodies [34, 35], occurs in a significant portion of MM patients afflicted with COVID-19. Various agents including but not limited to anti-IL-6 [36] monoclonal antibodies and JAK inhibitors [37] are presently under investigation to address potential components of immune dysregulation in COVID-19. We noted that patients who died from COVID-19 had alarmingly elevated D-dimer levels compared to survivors (median of $18.24 \mathrm{mg} / \mathrm{L}$ vs $1.96 \mathrm{mg} / \mathrm{L}$ ). Emerging research suggests that the overwhelming immune activation during SARS-CoV-2 infection is a potent catalyst for significant arterial and venous thromboembolism leading to strokes and pulmonary emboli [15, 38], and serum pro-inflammatory cytokines including IL-1 $\beta$, TNF- $\alpha$, and IL- 6 have been tied to endothelial damage underlying thrombus formation seen in COVID-19 [39]. To counter this possibility, a large majority of patients admitted to our institution in this cohort received therapeutic anticoagulation and none suffered bleeding events. Our data regarding inflammatory markers raises the question if the process driving severe D-dimer elevation in MM patients with COVID-19 is the same or is separate from the CRS-like process seen in many COVID-19 patients. 
Data on the persistence of SARS-CoV-2 by PCR and development of specific antibody response to the virus in potentially immunocompromised cancer patients have thus far been lacking. A significant majority of tested patients among this cohort cleared infection by PCR and developed antibodies despite a very high proportion of patients who fit the definition of classical myeloma-associated immunoparesis. Immunoparesis alone was not significantly associated with hospitalization or mortality and importantly did not appear to affect the development of anti-SARS-CoV-2 antibodies. Looking forward, we will need to determine if the development of antibodies confers protection against reinfection.

This study has the limitations of single institution, retrospective reporting of a smaller cohort of patients. Serological data were not available for a minority of the patients who were hospitalized at outside institutions. The observations reported here have to be confirmed by a larger series of data collected from multiple institutions and such efforts are underway. Few patients received COVID-19-directed treatment on clinical trials. The role of recently emergency approved anti-viral agent remdesivir, or convalescent plasma should be explored in the high-risk population with myeloma.

\section{Conclusions}

In this study of patients treated for myeloma at the Mount Sinai Hospital, we provide a detailed analysis of a cohort of $58 \mathrm{MM}$ and SMM patients who developed COVID-19. Although several demographic factors and comorbidities increased the risk of hospitalization and mortality, myeloma therapy and immunoparesis did not influence outcomes. In fact, survival was comparable to the overall population of New York during the pandemic, and patients generally mounted a significant antibody response to SARS-CoV-2. The data herein supports the need to maintain proactive management of MM patients by balancing their need for therapy with the increased risk of hospitalization and death in a subset of MM patients with COVID-19.

\section{Supplementary information}

Supplementary information accompanies this paper at https://doi.org/10. 1186/s13045-020-00934-X.

Additional file 1: Figure S1. Evolution of selected inflammatory biomarkers in a subset of patients $(n=12)$ hospitalized at the Mount Sinai Hospital for which the data was available. Different measurements from the same patient are connected. A linear regression line is plotted for the subgroup of patients that survived (blue, $n=8$ ) and died (red, $n$ $=4$ ), respectively.

\section{Abbreviations}

ACE: Angiotensin-converting enzyme; ALT: Alanine aminotransferase; ARB: Angiotensin II receptor blocker; ASCT: Autologous stem cell transplant; AST: Aspartate aminotransferase; BiPAP: Bi-level positive airway pressure;
BMI: Body mass index; bpm: Beats per minute; CAD: Coronary artery disease; CAR: Chimeric antigen receptor; CHF: Congestive heart failure; CKD: Chronic kidney disease; COPD: Chronic obstructive lung disease; COVID-

19: Coronavirus disease 2019; CPAP: Continuous positive airway pressure; CR: Complete response; CRS: Cytokine release syndrome; CRP: C-reactive protein; DOAC: Direct oral anticoagulant; ECOG: Eastern Cooperative Oncology Group; eGFR: Estimated glomerular filtration rate; ELISA: Enzymelinked immunosorbent assay; G-CSF: Granulocyte colony-stimulating factor; HIPAA: Health Insurance Portability and Accountability Act;

IMWG: International Myeloma Working Group; ICU: Intensive care unit; IL: Interleukin; IMiD: Immunomodulatory drug; IRB: Institutional review board; IQR: Interquartile range; ISS: International staging system; LDH: Lactate dehydrogenase; MM: Multiple myeloma; OR: Odd's ratio; PCR: Polymerase chain reaction; PD: Progressive disease; PI: Proteasome inhibitor; PR: Partial response; SARS-CoV-2: Severe acute respiratory syndrome coronavirus 2; sCR: Stringent complete response; SD: Stable disease; SMM: Smoldering multiple myeloma; TNF-a: Tumor necrosis factor-a; VGPR: Very good partial response

\section{Acknowledgements}

We would like to acknowledge all the staff and families for the selfless efforts in caring for patients who developed COVID-19, and the strength and courage of all patients affected by the pandemic.

\section{Authors' contributions}

Conceptualization, BW, OVO, THM, SJ, SP, and DM; Methodology, OVO; Investigation, BW, OVO, THM, DV, and SG; Writing-original draft, BW, OVO, THM; Writing - review \& editing, all authors; Funding acquisition, SJ.; Resources, MM, SJ, SP, and DM; Supervision, MM, SJ, SP, and DM. The authors read and approved the final manuscript.

Funding

There is no outside funding declared for this study.

\section{Availability of data and materials}

The datasets analyzed during the current study are not publicly available due to the US Federal Health Insurance Portability and Accountability Act (HIPAA) compliance, but a de-identified dataset may be available from the corresponding author on reasonable request.

\section{Ethics approval and consent to participate}

This study was performed in accordance with the Declaration of Helsinki and International Conference on Harmonization Guidelines for Good Clinical Practice (IRB: GCO\#: 11-1433).

\section{Consent for publication}

Not applicable.

\section{Competing interests}

A.C.: Advisory board and consulting fees from Amgen, Antegene, Celgene, Janssen, Karyopharm, Millennium/Takeda, Novartis Pharmaceuticals, Oncopeptides, Sanofi; research funding from Amgen, Celgene, Janssen, Millennium/Takeda, Novartis Pharmaceuticals, Pharmacyclics. S. J.: Advisory board and consulting fees from Celgene, Bristol-Myers Squibb, Janssen Pharmaceuticals and Merck. H. J. C: Employed by the Multiple Myeloma Research Foundation, advisory board and consulting fees from Genetech, Celgene, Bristol Myers Squibb, GlaxoSmithKline and received research funding from Takeda, Celgene, and Genetech. D. M.: Advisory board and consulting fees from Janssen, Celgene, Bristol Myers Squibb, Takeda, Legend, GlaxoSmithKline, Kinevant, and Foundation Medicine. B.W.: Consulting fees from Sanofi Genzyme. J. R.: Speaking fees from Celgene and Janssen, advisory board and consulting fees from Celgene, Janssen, Bristol Myers Squibb, Oncopeptides, Adaptive Biotechnologies, X4 Pharmaceuticals, Karyopharm, and Antegene. S. P.: Consulting fees from Foundation Medicine, research funding from Celgene and Karyopharm, supported by 1R01CA244899-01A1. All other authors declare no potential conflict of interest.

\section{Author details}

${ }^{1}$ Tisch Cancer Institute, Icahn School of Medicine at Mount Sinai, 10 East 102nd Street, 6th Floor, New York, NY 10029, USA. ²Department of Medicine, Icahn School of Medicine at Mount Sinai, New York, NY 10029, USA. 
${ }^{3}$ Precision Immunology Institute, Icahn School of Medicine at Mount Sinai, New York, NY 10029, USA.

Received: 31 May 2020 Accepted: 3 July 2020

Published online: 14 July 2020

\section{References}

1. Richardson S, Hirsch JS, Narasimhan M, et al. Presenting characteristics, comorbidities, and outcomes among 5700 patients hospitalized with COVID-19 in the New York City area. JAMA. 2020.

2. Robilotti EV, Babady NE, Mead PA, et al. Determinants of severity in cancer patients with COVID-19 illness. medRxiv. 2020; 2020.05.04.20086322.

3. Dai M, Liu D, Liu M, et al. Patients with cancer appear more vulnerable to SARS-COV-2: a multicenter study during the COVID-19 outbreak. Cancer Discov. 2020.

4. Liang W, Guan W, Chen R, et al. Cancer patients in SARS-CoV-2 infection: a nationwide analysis in China. Lancet Oncol. 2020;21:335-7.

5. Yu J, Ouyang W, Chua MLK, Xie C. SARS-CoV-2 transmission in patients with cancer at a tertiary care hospital in Wuhan, China. JAMA Oncol. 2020.

6. Zhang L, Zhu F, Xie L, et al. Clinical characteristics of COVID-19-infected cancer patients: a retrospective case study in three hospitals within Wuhan, China. Ann Oncol. 2020.

7. Kyle RA, Rajkumar SV. Multiple myeloma. Blood. 2008;111:2962-72.

8. Lee SJ, Borrello I. Role of the immune response in disease progression and therapy in multiple myeloma. Cancer Treat Res. 2016;169:207-25.

9. Kumar SK, Anderson KC. Immune therapies in multiple myeloma. Clin Cancer Res. 2016;22:5453-60.

10. Ingraham NE, Lotfi-Emran S, Thielen BK, et al. Immunomodulation in COVID19. Lancet Respir Med.

11. Mehta P, McAuley DF, Brown M, et al. COVID-19: consider cytokine storm syndromes and immunosuppression. Lancet. 2020.

12. Merad M, Martin JC. Pathological inflammation in patients with COVID-19: a key role for monocytes and macrophages. Nat Rev Immunol. 2020.

13. Liu D, Zhao J. Cytokine release syndrome: grading, modeling, and new therapy. J Hematol Oncol. 2018;11:121.

14. Lee DW, Santomasso BD, Locke FL, et al. ASTCT Consensus grading for cytokine release syndrome and neurologic toxicity associated with immune effector cells. Biol Blood Marrow Transplant. 2019;25:625-38.

15. Zhang C, Wu Z, Li J-W, Zhao H, Wang G-Q. The cytokine release syndrome (CRS) of severe COVID-19 and interleukin-6 receptor (IL-6R) antagonist Tocilizumab may be the key to reduce the mortality. Int J Antimicrob Agents. 2020;105954

16. Mehta P, McAuley DF, Brown M, Sanchez E, Tattersall RS, Manson JJ. COVID19: consider cytokine storm syndromes and immunosuppression. Lancet (London, England). 2020;395:1033-4.

17. Huang C, Wang Y, Li X, et al. Clinical features of patients infected with 2019 novel coronavirus in Wuhan, China. Lancet (London, England). 2020;395: 497-506.

18. Gong J, Dong H, Xia SQ, et al. Correlation analysis between disease severity and inflammation-related parameters in patients with COVID-19 pneumonia. medRxiv. 2020; 2020.02.25.20025643.

19. Rajkumar SV, Dimopoulos MA, Palumbo A, et al. International Myeloma Working Group updated criteria for the diagnosis of multiple myeloma. Lancet Oncol. 2014;15:e538-48.

20. Kumar S, Paiva B, Anderson KC, et al. International Myeloma Working Group consensus criteria for response and minimal residual disease assessment in multiple myeloma. Lancet Oncol. 2016;17:e328-e46.

21. Sørrig R, Klausen TW, Salomo M, et al. Smoldering multiple myeloma risk factors for progression: a Danish population-based cohort study. Eur J Haematol. 2016;97:303-9.

22. Widman DG, Gornisiewicz S, Shacham S, Tamir S. In vitro toxicity and efficacy of verdinexor, an exportin 1 inhibitor, on opportunistic viruses affecting immunocompromised individuals. PLoS One. 2018;13:e0200043.

23. Wu M, Gui H, Feng Z, et al. KPT-330, a potent and selective CRM1 inhibitor, exhibits anti-inflammation effects and protection against sepsis. Biochem Biophys Res Commun. 2018;503:1773-9.

24. Al Saleh AS, Sher T, Gertz MA. Multiple myeloma in the time of COVID-19. Acta Haematol. 2020:1-7.

25. Society IM. Recommendations for the management of myeloma patients during the COVID-19 pandemic; 2020.
26. Malard F, Mohty M. Management of patients with multiple myeloma during the COVID-19 pandemic. Lancet Haematol. 2020.

27. COVID-19: Data. 2020. at https://www1.nyc.gov/site/doh/covid/covid-19data.page.).

28. Cook G, Ashcroft AJ, Pratt G, et al. Real-world assessment of the clinical impact of symptomatic infection with severe acute respiratory syndrome coronavirus (COVID-19 disease) in patients with Multiple Myeloma receiving systemic anti-cancer therapy. Br J Haematol.

29. Gross CP, Essien UR, Pasha S, Gross JR, Wang S-Y, Nunez-Smith M. Racial and ethnic disparities in population level COVID-19 mortality. medRxiv. 2020; 2020.05.07.20094250.

30. Henning-Smith C, Tuttle M, Kozhimannil KB. Unequal distribution of COVID19 risk among rural residents by race and ethnicity. J Rural Health. 2020.

31. Howard G, Safford MM, Moy CS, et al. Racial differences in the incidence of cardiovascular risk factors in older Black and White adults. J Am Geriatr Soc. 2017:65:83-90.

32. Diane Marie Del Valle M, Seunghee Kim-Schulze PD, Hsin-Hui Huang PD, et al. An inflammatory cytokine signature helps predict COVID-19 severity and death. MEDRXIV. 2020;115758.

33. Jatiani SS, Aleman A, Madduri D, et al. Myeloma CAR-T CRS management with IL-1R antagonist anakinra. Clin Lymphoma MyelomaLeukemia. 2020.

34. Dai H, Wu Z, Jia H, et al. Bispecific CAR-T cells targeting both CD19 and CD22 for therapy of adults with relapsed or refractory $B$ cell acute lymphoblastic leukemia. J Hematol Oncol. 2020;13:30.

35. Costa $L J$ WS, Bermúdez A. First clinical study of the B-cell maturation antigen (BCMA) $2+1$ T cell engager (TCE) CC-93269 in patients (pts) with relapsed/refractory multiple myeloma (RRMM): interim results of a phase 1 multicenter trial. Abstract \#143. American Society of Hematology. Orlando: ASH Annual Meeting Proceedings; 2019.

36. Xu X, Han M, Li T, et al. Effective treatment of severe COVID-19 patients with tocilizumab. Proc Natl Acad Sci. 2020;117:10970-5.

37. Cao $Y$, Wei J, Zou L, et al. Ruxolitinib in treatment of severe coronavirus disease 2019 (COVID-19): A multicenter, single-blind, randomized controlled trial. J Allergy Clin Immunol. 2020.

38. Tseng C-TK, Perrone LA, Zhu H, Makino S, Peters CJ. Severe acute respiratory syndrome and the innate immune responses: modulation of effector cell function without productive infection. J Immunol. 2005:174:7977-85.

39. Bryce C, Grimes Z, Pujadas E, et al. Pathophysiology of SARS-CoV-2: targeting of endothelial cells renders a complex disease with thrombotic microangiopathy and aberrant immune response. The Mount Sinai COVID19 autopsy experience. medRxiv. 2020; 2020.05.18.20099960.

\section{Publisher's Note}

Springer Nature remains neutral with regard to jurisdictional claims in published maps and institutional affiliations.

Ready to submit your research? Choose BMC and benefit from:

- fast, convenient online submission

- thorough peer review by experienced researchers in your field

- rapid publication on acceptance

- support for research data, including large and complex data types

- gold Open Access which fosters wider collaboration and increased citations

- maximum visibility for your research: over $100 \mathrm{M}$ website views per year

At $\mathrm{BMC}$, research is always in progress.

Learn more biomedcentral.com/submissions 